\section{Inter- und Transdisziplinarität Herausforderung für die Nachhaltigkeitsforschung}

\author{
V. Rabelt, Th. Büttner, K.-H. Simon (Hg.): \\ Neue Wege in der Forschungspraxis. \\ Begleitinstrumente in der transdisziplinä- \\ ren Nachhaltigkeitsforschung. München: \\ ökom verlag, 2007, 122 S., ISBN 978-3- \\ 86581-015-1, € 24,80
}

\section{R. Defila, A. Di Giulio, M. Scheuermann: Forschungsverbundmanagement. Hand- buch für die Gestaltung inter- und trans- disziplinärer Projekte. Zürich: vdf Hoch- schulverlag AG an der ETH Zürich, 2006, 348 S., ISBN 978-3-7281-3042-6, $€$ 29,80}

\section{Rezension von Achim Daschkeit, Umweltbundesamt}

Viele Wissenschaftler finden Diskussionen über Inter- und Transdisziplinarität überflüssig oder notfalls als Thema für Sonntagsreden geeignet: Es werde dort über etwas geredet, was zwar prinzipiell sinnvoll sein mag, aber in der Realität und Praxis wissenschaftlichen Arbeitens und Forschens sei die disziplinäre Verortung entscheidend. Diese - etwas überspitzt formulierte - Perspektive steht in deutlichem Kontrast zu Debatten und Aktivitäten, die Inter- und Transdisziplinarität thematisieren und vor allem in den Bereichen Umwelt- und Nachhaltigkeitsforschung seit vielen Jahren voranbringen. Diese Thematisierung reicht heute von allgemeinen Erörterungen zu ganz konkreten Empfehlungen, die helfen sollen, den inter- und transdisziplinären Forschungstyp zu bewerten und dessen Projektmanagement zu befördern.

Vor diesem Hintergrund sind die beiden hier rezensierten Bücher zwar grundverschieden, passen aber dennoch (oder vielleicht gerade deswegen) sehr gut zueinander.

\section{Neue Wege in der Forschungspraxis}

Das Buch, das von Vera Rabelt, Thomas Büttner und Karl-Heinz Simon herausgegeben wurde, beschreibt Erfahrungen aus dem Förderschwerpunkt des Bundesministeriums für Bildung und
Forschung „Möglichkeiten und Grenzen neuer Nutzungsstrategien - Regionale Ansätze“. Die Koordination der Projekte / Projektverbünde innerhalb des Förderschwerpunktes wurde vom Umweltbundesamt ausgeführt. Ein Element der Koordinationstätigkeiten bestand in der Einrichtung einer Arbeitsgruppe „Transdisziplinarität“, die der Frage nachging, inwieweit spezielle Begleitinstrumente zur inhaltlichen wie sozialen Integration der Projektverbünde bzw. des Förderschwerpunktes nützlich sind. Im Buch „Neue Wege in der Forschungspraxis. Begleitinstrumente in der transdisziplinären Nachhaltigkeitsforschung" werden als Ergebnis verschiedene Ansätze vorgestellt wie etwa Supervision, Coaching, Organisationsberatung, Monitoring, Moderation und Kooperationsmanagement. Die Beschreibung dieser Instrumente erfolgt sowohl in allgemeiner Form als auch in Bezug auf die konkret durchgeführten Projekte. Generelles Ziel des Einsatzes dieser Instrumente sei es, die Ergebnisqualität der Projektverbünde zu verbessern - jedenfalls ist das eine aus meiner Sicht naheliegende Interpretation, die sich aber in dieser konkreten Formulierung im Buch nicht findet.

Nach Erläuterungen zum Förderschwerpunkt, zur transdisziplinären Nachhaltigkeitsforschung, sowie zur oben erwähnten Arbeitsgruppe und zu den Anforderungen an die Umsetzung transdisziplinärer Forschung werden die genannten Instrumente sowohl für die Projekt- als auch für die Förderschwerpunktebene vorgestellt. Diese differenzierte Betrachtung ist sinnvoll, da neben einer projektinternen Integration auch eine projektübergreifende Integration sinnvoll sein kann. Dem Buch liegt die Annahme zugrunde, dass eine Unterstützung der sozialen Integration heterogener Projektbeteiligter (Wissenschaftler, Praxispartner) gleichzeitig ein Beitrag zur - inhaltlichen - Wissensintegration leiste. Die soziale Integration steht im Mittelpunkt, die inhaltliche Integration wird weniger ausführlich betrachtet bzw. es wird nicht nachvollziehbar gezeigt, wie soziale Integration (also die Begleitinstrumente) die kognitive Integration konkret unterstützt. Wollte man das im Detail (womöglich noch empirisch) zeigen, würde man den Anspruch des Buches überfrachten. Konkret hieße das nämlich, den „Nutzen“ der Begleitinstrumente zu ermitteln (S. 37); diesen Anspruch ernst zu nehmen, wäre ein eigenes, weiteres 
Projekt gewesen, weil es m. E. keineswegs trivial ist, solch einen Nutzen zu ermitteln. In diesem Punkt sind Anspruch und Wirklichkeit des Buches nicht ganz kongruent, was dem Nutzen des Buches (Einführung in Begleitinstrumente und die Dokumentation praktischer Erfahrungen) keinen Abbruch tut.

Man kann im Sinne eines Zwischenfazits festhalten, dass der Spagat zwischen dem Einsatz von Begleitinstrumenten für die Projekte und Projektverbünde im Förderschwerpunkt und der aktiven Steuerung der Verbünde (in dem Sinn, dass ein definiertes Ziel mit den Projekten bzw. dem Förderschwerpunkt insgesamt erreicht werden soll) nicht ganz unproblematisch ist. Dennoch ist das Buch sehr wertvoll, weil es die umfänglichen Bemühungen beinhaltet, den Aspekt der ,sozialen Integration“ überhaupt einmal zentral aufzugreifen und hierfür erfahrungsgesättigte Beispiele darzustellen.

\section{Forschungsverbundmanagement}

Einen ganz anderen Zugang zur Thematik wie inter- und transdisziplinäre Forschung unterstützt werden könne, haben Rico Defila, Antonietta Di Giulio und Michael Scheuermann gewählt. Ihr Ansatz, in dem das Forschungsverbundmanagement im Mittelpunkt steht, verträgt sich ideal mit dem von Vera Rabelt et al. Die Autoren gehen davon aus, dass Forschungsverbundmanagement als eigenständige, anspruchsvolle wissenschaftliche Aufgabe angesehen werden sollte. Ihr Buch „Forschungsverbundmanagement. Handbuch für die Gestaltung inter- und transdisziplinärer Projekte" soll Hinweise für das Management inter- und transdisziplinärer Verbünde geben im Sinne von Werkzeugen für Managementverantwortliche, die zuständig sind für Koordination, Vernetzung, Initiierung und Moderation inter- und transdisziplinärer Zusammenarbeit. Die Autoren haben den Anspruch, die gesamte Laufzeit des Vorhabens - also von der Planung bis zum Abschluss - zu beschreiben.

In dem sehr hübsch illustrierten und vom Layout her ansprechenden Werk geht es um die Grundlagen des Forschungsverbundmanagements. Zweckmäßigerweise werden allgemeine Ausführungen zum Management, zu Inter- und Transdisziplinarität, konkreter dann zum For- schungsverbundmanagement im eigentlichen Sinne gemacht. All das mündet in der konkreten Formulierung und Darstellung einer AufgabenMatrix, die auch als Beilage dem Buch beiliegt. Die Intention der Autoren ist klar und verständlich: Man nehme die Beilage, hänge sie sich über den Schreibtisch, sodass man jeden Tag die Aufgaben vor Augen hat. Es kann tatsächlich sehr hilfreich sein, die Aufgaben-Matrix oft zurate zu ziehen, denn sie erleichtert die Strukturierung und erinnert an Dinge, die anderweitig leicht vergessen werden können.

Die vielen Tipps und Hinweise zum Management inter- und transdisziplinärer Forschungsverbünde sind in Aufgabenpakete differenziert: Gemeinsame Ziele und Fragen, Vernetzung der Forschungsarbeiten, Synthesebildung, Entwicklung gemeinsamer Produkte, Auswahl der Personen und Teamentwicklung, Beteiligung Externer, interne und externe Kommunikation und Organisation der Arbeit. Die Struktur der Darstellung innerhalb der Aufgabenpakete ist dann immer gleich: Es gibt jeweils eine kurze Einführung, eine kurze Beschreibung der Aufgaben in diesem Paket sowie jede Menge praktischer Tipps. Die Darstellung ist unterteilt in die üblichen Phasen eines Forschungsprojektes (Vorbereitung, Start, Durchführung, Abschluss) und zum Schluss wird auf allfällige Gefahren verwiesen. Im Anhang des Buches finden sich Angaben zur sogenannten DACH-Erhebung: Empirisch wurden in 4 Forschungsprogrammen in Deutschland, Österreich und der Schweiz Angaben zum Forschungsverbundmanagement erhoben. Hier findet man also den eigentlichen Ursprung des Buches: Nach Auswertung der Erhebung wurde erkennbar, dass eine praktisch orientierte Anleitung zum Management von Forschungsverbünden fehlte. Die Ergebnisse der DACH-Erhebung sind die empirische Grundlage für die vielen Tipps und Hinweise.

Aus meiner persönlichen Erfahrung heraus sind die Arbeitspakete „Gemeinsame Ziele und Fragen“ und „Synthesebildung" von besonderer Relevanz und Schwierigkeit. Was in der Vorphase bzw. Planung nicht oder unzureichend geregelt ist - beispielsweise die unzureichend detaillierte Planung von Integration und Synthese -, lässt sich im Projektverlauf nur schwerlich korrigieren. Diesem Aufgabenpaket möchte ich mich im Folgenden etwas eingehender zuwenden. 
Völlig zu recht schreiben die Autoren, dass die Synthese (im Sinne inhaltlicher Synthese / Integration; ich verwende diese beiden Begriffe hier synonym) eines Forschungsverbundes von Anfang an bedacht und geplant werden muss. Somit ist die Beschreibung des gemeinsamen Forschungsgegenstandes (im Zuge der Projektplanung) bereits eine erste wichtige Integrationsleistung. Die Autoren sagen es deutlich: Ohne Synthese habe die Arbeit eines Forschungsverbundes keinen Mehrwert.

Gleichzeitig meint Synthese nicht lediglich die Zusammenführung der Ergebnisse von Einzelprojekten, sondern mehr - an dieser zentralen Stelle schweigen sich die Autoren ein wenig aus: Was ist denn nun eigentlich dieses „mehr"? Ist es immer erkennbar? Integration wird im Buch als Synthese mit geeigneten Methoden und Verfahren umschrieben - genau, Zustimmung. Hier hätte sich ein Beispiel gut gemacht, damit der Leser einen Eindruck davon bekommt, was das Ergebnis von Integration sein könnte. Rico Defila et al. schreiben, die gängigen Syntheseverfahren würden nicht von allein den Erfolg der Synthesebildung gewährleisten - auch hier stimme ich zu, aber was sind die ,gängigen Syntheseverfahren“? Erwähnt werden Modellierungstechniken, die Nutzung von Szenariotechniken, systemtheoretische Ansätze. Eher „nebenbei“ (S. 144) sind weitere Methoden wie Thesen, Handlungsempfehlungen, Szenarien angeführt. Auch werden Methoden der Synthesebildung beschrieben, wobei die Methodentypen durch ein Beispiel hätten konkretisiert werden können. Erwähnt wird auch die Orientierung an Produkten - es bleibt aber auch hier ein wenig unklar, was unter „Produkten“ verstanden werden kann. Die Autoren beschreiben überdies, dass Synthese durch Managementverantwortliche in erster Linie Moderation bedeutet (s. o. das Buch von Vera Rabelt et al.); inwieweit hier auch eine inhaltliche Synthese erarbeitet werden soll, ist eine viel diskutierte Frage, weil eine gewisse Interessenkollision zwischen inhaltlicher Arbeit (Synthese) und neutraler Moderationstätigkeit droht. Auch wenn dieses Buch die eine oder andere Detailfrage offenlassen muss, ist es gerade für die Praktiker des Forschungsverbundmanagements quasi unverzichtbar.

\section{Fazit}

Nimmt man die beiden hier vorgestellten Bücher zusammen, erhält man einen vielfältigen und umfassenden Überblick über den derzeitigen Stand der Diskussion zu Inter- und Transdisziplinarität. Jedes Buch für sich allein kann die vielfältigen Aspekte der Diskussion um Interund Transdisziplinarität nicht umfassend darstellen; nimmt man beide Bücher gemeinsam, erhält man bereits einen weit reichenden Einblick in den Stand der Debatte. Was beiden Büchern fehlt und wohl einer weiteren Publikation vorbehalten bleibt, ist eine detaillierte Auseinandersetzung mit Good-Practise-Beispielen von Interund Transdisziplinarität, die in erster Linie auf die Möglichkeiten und Grenzen integrativer Methoden und Techniken fokussiert.

\section{$《 》$}

\section{Industrial Ecology als Chance für zukunftsorientiertes Wirtschaften}

\author{
Isenmann, R., von Hauff, M. (Hg.): Indus- \\ trial Ecology: Mit Ökologie zukunftsori- \\ entiert wirtschaften. München: Spektrum \\ Akademischer Verlag, 2007, 326 S., ISBN \\ 978-3-8274-1806-7, $€$ 49,50
}

\section{Rezension von Witold-Roger Poganietz, ITAS-ZTS}

Das Forschungsgebiet „Industrial Ecology“ ist, auch wenn die Wurzeln weit in die 1950er Jahre zurück reichen, ein relativ junger Zweig der Umweltwissenschaften (Erkman, S. 32). Trotz oder gerade aufgrund - ihrer globalen Ausrichtung, dominieren englischsprachige Publikationen den wissenschaftlichen Diskurs, während deutschsprachige Studien kaum ihr Publikum finden. Die Fokussierung auf englischsprachige Arbeiten erschwert den breiten Zugang zur Industrial Ecology. Um den Zugang für Forscher und Praktiker, Lehrende und Lernende aus dem deutschsprachigen Raum zu erleichtern, haben Ralf Isenmann und Michael von Hauff den Sammelband „Industrial Ecology: Mit Ökologie 